(C) EDP Sciences, 2009

DOI: 10.1051 /orvie/2009004

\title{
Self organization and evolution in mathematical models
}

\author{
M. Thieullen \\ Laboratoire de Probabilités et Modèles Aléatoires, Boîte 188, Université \\ Paris VI, 4 place Jussieu, 75252 Paris Cedex 05, France \\ e-mail: michele.thieullen@upmc.fr
}

\begin{abstract}
Through several examples we illustrate to non mathematicians how biological situations can be translated in mathematical terms. Even simple mathematical models can be very efficient to clarify a complex biological situation and the same basic mathematical procedures can be found in different biological frameworks. In particular stability analysis under small fluctuations of non linear models possessing different time scales is fundamental, especially bifurcation properties. An important issue is also to choose between deterministic and stochastic models. We illustrate these points through classical examples coming from the formation of patterns (morphogenesis), vision (visual hallucinations), onset of periodic oscillations in the neuronal activity due to random noise and a mathematically related example of stochastic resonance.
\end{abstract}

\section{INTRODUCTION}

Our presentation in the present paper is primarily directed towards non mathematicians. Through several examples we would like to show how biological situations can be translated in mathematical terms. Mathematical models have at least two advantages: they help exhibiting universal properties commun to a priori very different situations, and also they enable identifying the relevant parameters among the available collection of parameters describing a system which may be quite large. We want to stress that even simple mathematical models can be very efficient to clarify a complex biological situation. In particular the stability analysis under small fluctuations of non linear models possessing different time scales is fundamental, especially their bifurcation properties. An important issue is also to choose between deterministic and stochastic models. We will illustrate these points through classical examples coming from the formation of patterns (morphogenesis), vision (visual hallucinations), onset of periodic oscillations in the neuronal activity due to random noise and a mathematically related example of stochastic resonance. Our main reference for deterministic models is Murray's book [9] on mathematical modelling in biology.

\section{MORPHOGENESIS}

The mathematical modeling of morphogenesis was iniated by Turing who created the notion of diffusion driven instability. The formation of patterns is a nonlinear phenomenon. However a good indication of the possible patterns is provided by the analysis of the linear stability. In particular the linear analysis gives the range

Article available at http://www.origins-and-evolution.org or http://dx.doi.org/10.1051/orvie/2009004 
of parameters which allow the formation of patterns. The mathematical model of morphogenesis is a system of nonlinear reaction-diffusion equations. The first step is to exhibit dimensionless parameters. In dimensionless parameters this system takes the following form

$$
\begin{aligned}
& \partial_{t} u=\gamma f(u, v)+\Delta u \\
& \partial_{t} v=\gamma g(u, v)+d \Delta v
\end{aligned}
$$

$u(x, t), v(x, t)$ are for instance the concentrations of two chemicals. The kinetics of the two components or species given by $f$ and $g$ are known in general as well as $d$. Parameter $\gamma$ is related to the size of the considered embryonic domain. The domain in which the space time variable $(t, x)$ evolves as well as initial and boundary conditions must be specified. Bounded versus infinite domains $B$ for the spatial variable makes a difference as we will see. This is a model of self-organisation, which means that the system receives no external input, if the relevant boundary condition expresses that there is no flux through the boundary: $(n \cdot \nabla u)=0$ on $\partial B$ where $\partial B$ is the boundary of $B$ and $n$ is the normal vector at each point of $\partial B$.

The possibility that patterns appear corresponds to a discussion in the $(d, \gamma)$ parameter space. More precisely the first step is to consider the equilibrium points of the system without diffusion and its linear stability. Patterns will appear if such an equilibrium point is stable to small perturbations in the absence of diffusion but unstable to small spatial perturbations in presence of diffusion. Patterns are identified with eigenfunctions. Let us recall that for a system of the form

$$
\begin{aligned}
& \frac{d u}{d t}=\gamma f(u, v) \\
& \frac{d v}{d t}=\gamma g(u, v)
\end{aligned}
$$

equilibrium points are the points $\left(u_{0}, v_{0}\right)$ such that $f\left(u_{0}, v_{0}\right)=g\left(u_{0}, v_{0}\right)=0$.

\subsection{Linear stability analysis in a bounded domain}

Linearizing around $\left(u_{0}, v_{0}\right)$ means that one considers that $w=\left(u-u_{0}, v-v_{0}\right)$ is a small perturbation, for example that it depends on a small parameter $\epsilon$ and is given by $\epsilon f_{1}+\epsilon^{2} f_{2}+\epsilon^{3} f_{3}+\cdots$ and keeps only terms of order 1 in $\epsilon$. In particular, the perturbation satisfies linear conditions. When the domain $B$ is bounded we thus have to look for $w(t, x)=\Sigma_{k} c_{k} \mathrm{e}^{\lambda t} W_{k}(x)$ satisfying simultaneously

$$
\begin{gathered}
\partial_{t} w=\gamma A w+D \nabla^{2} w \\
\nabla^{2} W_{k}+k^{2} W_{k}=0
\end{gathered}
$$

where $A:=\left[\begin{array}{ll}f_{u} & f_{v} \\ g_{u} & g_{v}\end{array}\right], D:=\left[\begin{array}{ll}1 & 0 \\ 0 & d\end{array}\right] .(2.2)$ is called a spatial eigenvalue problem, $W_{k}$ are the eigenfunctions. As a consequence of (2.1)-(2.2) the parameters $\lambda$ and $k$ are not 
independant: $W_{k}$ must be non trivial and satisfy

$$
\lambda W_{k}=\left(\gamma A-k^{2} D\right) W_{k}
$$

The possibility to generate patterns corresponds to the existence of pairs $(k, \lambda(k))$ such that

- the determinant of the matrix $\lambda(k) I-\gamma A+k^{2} D$ is equal to 0 so that a non trivial $W_{k}$ exists

- $\operatorname{Re} \lambda(0)<0$ which corresponds to the condition of stability without diffusion

- $\operatorname{Re} \lambda(k)>0$ for some $k$ which corresponds to the condition of instability in presence of diffusion

More precisely, let us denote by $\lambda_{1}(k)$ and $\lambda_{2}(k)$ the two eigenvalues of $\lambda(k) I-\gamma A+$ $k^{2} D$. When $k=0$ the real parts of $\lambda_{1}(0)$ and $\lambda_{2}(0)$ (the eigenvalues of $A$ ), are strictly negative if and only if

- $\lambda_{1}(0)+\lambda_{2}(0)=f_{u}+g_{v}<0$

- $\lambda_{1}(0) \lambda_{2}(0)=f_{u} g_{v}-f_{v} g_{u}>0$

For general $k$, the determinant of $\lambda(k) I-\gamma A+k^{2} D$ is the second order polynomial $\lambda^{2}+\lambda\left(k^{2}(1+d)-\gamma\left(f_{u}+g_{v}\right)\right)+p\left(k^{2}\right)$ where $p\left(k^{2}\right)=d k^{4}-\gamma\left(d f_{u}+g_{v}\right) k^{2}+\gamma^{2} \operatorname{det} A$. The sum of its solutions is $\lambda_{1}(k)+\lambda_{2}(k)=\gamma\left(f_{u}+g_{v}\right)-k^{2}(1+d)$. If the previous conditions on $\lambda(0)$ are satisfied, this sum remains strictly negative for any $k$. Therefore $\operatorname{Re} \lambda_{1}(k)>0$ or $\operatorname{Re} \lambda_{2}(k)>0$ is possible only if $\lambda_{1}(k)$ and $\lambda_{2}(k)$ have opposite sign that is if their product $\operatorname{Re} \lambda_{1}(k) R e \lambda_{2}(k)<0$. This product coincides with $p\left(k^{2}\right)$ which is an even function of $k$ going to infinity when $k^{2}$ goes to infinity. It may never take strictly negative values; it depends on parameter $d$. We are thus interested in the curves which represent $p\left(k^{2}\right)$ for each value of $d$ called dispersion curves. There exists a critical value of $d$ denoted by $d_{c}$ such that for $d<d_{c}$ the function $p\left(k^{2}\right)$ is always strictly positive; in this case no instability is observed in presence of diffusion, no Turing pattern is generated. When $d=d_{c}, p\left(k^{2}\right)$ fails to be strictly positive except for one value of $k^{2}$ denoted by $k_{c}^{2}$. For $d>d_{c}, p\left(k^{2}\right)$ is strictly negative for $\left.k^{2} \in\right] k_{1}^{2}, k_{2}^{2}\left[\right.$ an interval containing $k_{c}^{2}$. The size of this interval decreases with $\gamma$ : it tends to zero with $\gamma$. For $k$ close to $k_{c}$, the spatial patterns are approximately the solutions of the linear system. The value $k_{c}$ is also called a bifurcation value.

As an example let us consider (cf. [9])

$$
\begin{aligned}
& \partial_{t} A=F(A, B)+D_{A} \nabla^{2} A \\
& \partial_{t} B=G(A, B)+D_{B} \nabla^{2} B
\end{aligned}
$$

with $F(A, B)=k_{1}-k_{2} A+k_{3} A^{2} B$ and $G(A, B)=k_{4} A^{2}-k_{5} B$.

- Step 1: pass to dimensionless parameters.

$$
\begin{aligned}
& d=\frac{D_{B}}{D_{A}}, \quad \gamma=\frac{k_{2}}{D_{A}} \\
& a=\frac{k_{1}}{k_{2}}\left(\frac{k_{3}}{k_{2}}\right)^{\frac{1}{2}}, \quad b=\frac{k_{4}}{k_{2}}\left(\frac{k_{3}}{k_{2}}\right)^{\frac{1}{2}} \\
& u=A\left(\frac{k_{3}}{k_{2}}\right)^{\frac{1}{2}}, \quad v=B\left(\frac{k_{3}}{k_{2}}\right)^{\frac{1}{2}}, \quad \tilde{t}=D_{A} t .
\end{aligned}
$$


The new kinetics are given by $f(u, v)=a-u+u^{2} v$ and $g(u, v)=b-u^{2} v$. The equilibrium point: $\left(u_{0}, v_{0}\right)=\left(a+b, \frac{b}{(a+b)^{2}}\right)$

- Step 2: study in parameter space, dispersion curves.

The identity $\operatorname{det}\left(\lambda I-\gamma A+k^{2} D\right)=0$ becomes $\lambda^{2}+\alpha\left(k^{2}\right) \lambda+p\left(k^{2}\right)=0$ with

$$
\begin{aligned}
& \alpha\left(k^{2}\right)=k^{2}(1+d)-\gamma\left(\frac{b-a}{a+b}-(a+b)^{2}\right) \\
& p\left(k^{2}\right)=\gamma^{2}(a+b)^{2}-\gamma k^{2}\left(d \frac{b-a}{a+b}-(a+b)^{2}\right)+d k^{4}
\end{aligned}
$$

We want both

- (1) $\operatorname{Re} \lambda(0)<0$ which is equivalent to $\frac{b-a}{a+b}-(a+b)^{2}<0$

- (2) $\operatorname{Re} \lambda(k)>0$ for some $k$ which is equivalent to $p_{\min }<0$

In this case the dispersion curves are of the following form (notice the critical diffusion $d_{c}$ and critical wavenumber $k_{c}$ ).
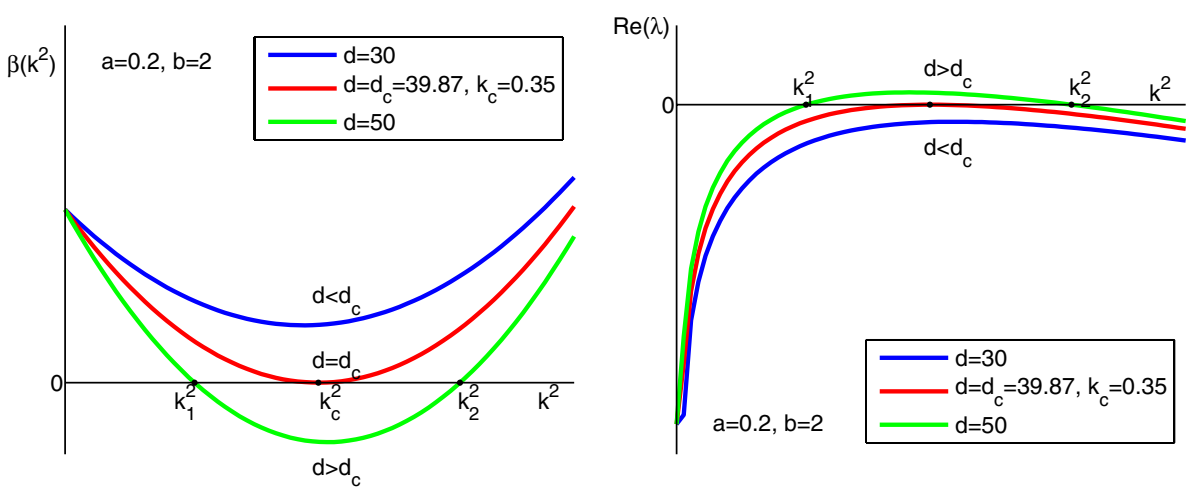

- Step 3: Spatial Eigenvalue Problem.

For a one dimensional $B=] 0, p$, the spatial eigenvalue problem is given by

$$
\begin{aligned}
W_{x x}+k^{2} W & =0, \quad x \in] 0, p[ \\
W_{x} & =0, \quad x=0, \quad x=p
\end{aligned}
$$

whose solutions are $W_{k}(x)=c_{n} \cos (k x)$ where $k=\frac{n \pi}{p}, n$ is an integer. Patterns exist if the admissible interval $] k_{1}^{2}, k_{2}^{2}$ [ provided by the dispersion curves is not empty. This is equivalent to the existence of integers $n$ satisfying a condition of the form

$$
\gamma L(a, b, d)<\left(\frac{n \pi}{p}\right)^{2}<\gamma M(a, b, d)
$$

Therefore no pattern exists if $\gamma$ is too small.

For 2-dimensional $B=] 0, p[\times] 0, q[$ the solutions of the spatial eigenvalue problem are the functions

$$
W_{k}(x, y)=\cos \left(\frac{n \pi x}{p}\right) \cos \left(\frac{m \pi y}{q}\right)
$$


with $n, m$ integers. $k^{2}=\pi^{2}\left(\frac{n^{2}}{p^{2}}+\frac{m^{2}}{q^{2}}\right)$ must satisfy $\gamma L(d)<k^{2}<\gamma M(d)$ which again has no solution if $\gamma$ is too small.

\section{VISUAL HALLUCINATIONS}

The previous linear stability method can be applied to a slightly different situation where not only short range diffusion is taken into account (as in reaction-diffusion systems) but also long range effects by means of integral equations instead of partial differential equations. This is an example of an unbounded domain $B$. Our reference for this section is a paper by Ermentrout and Cowan [3], [9].

Let us consider a collection of neurons located in a domain $B$; we denote by $n(t, z):=$ the firing rate of the neurons in position $z$ at time $t$. This rate is influenced by the neighbouring neurons (with the assumption that the further the neighbour, the lesser the influence). This is expressed thanks to a spatial kernel $K\left(z, z^{\prime}\right)$; in many instances one assumes that this kernel depends only on the distance between the neighbours and tends to zero when the distance goes to infinity: $K\left(z, z^{\prime}\right)=K\left(\left|z-z^{\prime}\right|\right)$ with $\lim _{+\infty} K=0$. The following integral equation describes the evolution of the firing rate

$$
n_{t}(t, z)=f(n)+\int_{B} K\left(z-z^{\prime}\right)\left(n\left(t, z^{\prime}\right)-n_{0}\right) d z^{\prime}=f(n)+K \star\left(n-n_{0}\right)
$$

if we assume that without neighborhood influence its time evolution is given by $n_{t}=f(n)$ for which $n=n_{0}$ is an equilibrium point (an autonomous firing rate).

The mathematical description of observed visual hallucinations can be fitted in this general framework after some adjustments that we describe briefly. In their original study on this topic Ermentrout and Cowan [3] assume that cortical neurons are either excitatory or inhibitory and consider that neighborhood influence is decomposed into influence inside each class as well as cross influence of one class on the other one. They also consider coarse grained rates of the form $E(z, t)=\int_{-\infty}^{t} k(t-s) n_{E}(s, z) d s$ and $I(z, t)=\int_{-\infty}^{t} k(t-s) n_{I}(s, z) d s$ to incorporate time delays and decays through a time kernel $k$. Notations $E$ and $I$ stands for excitatory and inhibitory. Then Ermentrout and Cowan's model takes the form

$$
\begin{gathered}
\partial_{t} E=-E+S_{E}\left(\alpha_{E E} K_{E E} \star E-\alpha_{I E} K_{I E} \star I\right) \\
\partial_{t} I=-I+S_{I}\left(\alpha_{E I} K_{E I} \star E-\alpha_{I I} K_{I I} \star I\right)
\end{gathered}
$$

The domain $B$ is 2-dimensional. A possible spatial kernel is $\exp -b\left(x^{2}+y^{2}\right)$. The equilibrium point is $\left(E_{0}, I_{0}\right)=(0,0)$. As in the previous section one linearizes around $(0,0)$ and looks for solutions of the resulting linear system as

$$
(E, I)=V \exp \left[\lambda t+i\left(k_{1} x+k_{2} y\right)\right]
$$

The wave vector is $k=\left(k_{1}, k_{2}\right)$ with $k^{2}=k_{1}^{2}+k_{2}^{2}$. Close to bifurcation (for $k$ close to $k_{c}$ ) the patterns are given by linear combinations of the following functions: $\exp k_{c} x, \exp k_{c} y$, $\exp k_{c}(\cos \Phi x+\sin \Phi y)$ for some angle $\Phi$. The patterns for these basic functions are stripes as in Figure 1 below. Linear combinations of exponentials obtained for $\Phi= \pm \frac{\pi}{6}$ and $\Phi=\frac{\pi}{2}$ gives rise to patterns with hexagonal symmetry as in Figure 2 below. 

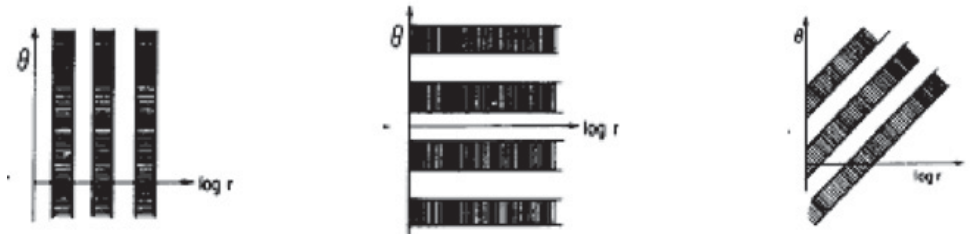

Figure 1. Stripes Patterns reproduced from [3].

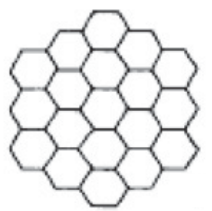

Figure 2. Hexagonal Patterns reproduced from [3].

\section{NOISE INDUCED OSCILLATIONS}

One of the simple models of the space-clamped electrical activity of a neuron is the FitzHugh-Nagumo System

$$
\begin{aligned}
\delta \dot{x}_{t} & =-y_{t}+f\left(x_{t}\right) \\
\dot{y}_{t} & =x_{t}-a
\end{aligned}
$$

where $x_{t}$ is the membrane potential (actually the potential difference accross the membrane), $f(x)=x(x-\theta)(1-x)$ where $0<\theta<1$. $\theta$ is the threshold. This 2-dimensional system is a simplified form of the more precise 4-dimensional model proposed by Hodgkin and Huxley which takes into account the flows of calcium, potassium and other leaky ions through the membrane, responsible for the electrical activity [8]. Passage from 2-dimensional to 4-dimensional system is meaningful thanks to an important property of these systems: different time scales are involved. Such systems are called slow-fast systems. In the FitzHugh-Nagumo system this is materialized by the fact that the parameter $\delta$ is small, variable $x$ is fast, while variable $y$ is slow. Indeed by the time change $s=\delta t$ one gets:

$$
\begin{aligned}
& \dot{\tilde{x}}_{s}=-\tilde{y}_{s}+f\left(\tilde{x}_{s}\right) \\
& \dot{\tilde{y}}_{s}=\delta\left(\tilde{x}_{s}-a\right)
\end{aligned}
$$

Periodic behaviour is possible for a range of parameters $a$ to be detailed below. We show that periodic oscillations can be generated for $a$ outside this range in the presence of noise, thus using a stochastic model rather than a deterministic one. These noise-induced oscillations can be seen as an example of stochastic resonance phenomenon. We will also illustrate briefly another situation where this phenomenon is relevant encountered 
in climate modeling. (4.1)-(4.2) is not the only possible form for a FitzHugh-Nagumo system, in particular one can consider the following one [6]

$$
\begin{aligned}
\delta \dot{x}_{t} & =-y_{t}+f\left(x_{t}\right)+I \\
\dot{y}_{t} & =\alpha x_{t}-\beta y_{t}
\end{aligned}
$$

where $I$ stands for the exterior current applied to the membrane. Under this form periodic behaviour is possible for some range of parameter $I$. The range of parameter $a$ which allow for oscillations in (4.1)-(4.2) is provided by the study of its linear stability. There is a unique equilibrium point $(a, f(a))$. Let us denote by $a_{0}<a_{1}$ the two reals such that $f^{\prime}\left(a_{0}\right)=f^{\prime}\left(a_{1}\right)=0$ where $f$ attains a minimum and a maximum. For $\left.a \in\right]-\infty, a_{0}$ [ or ] $a_{1},+\infty$ [ the equilibrium point $(a, f(a))$ is stable which roughly means that if the initial value $\left(x_{0}, y_{0}\right)$ is chosen close to $(a, f(a))$, the trajectory of $\left(x_{t}, y_{t}\right)$ remains in a neighborhood of this point and converges to it as $t$ goes to infinity. For $a \in] a_{0}, a_{1}$ [ the equilibrium point is unstable and limit cycle oscillations are possible. As in previous sections $a_{0}$ and $a_{1}$ are the bifurcation parameters since the stability radically changes as one passes through these values.

Let us study the linear stability of (4.1)-(4.2) around $a_{1}$ for instance, that is we consider

$$
\begin{aligned}
\delta \dot{x}_{t} & =-y_{t}+f\left(x_{t}\right) \\
\dot{y}_{t} & =x_{t}-\left(a_{1}+\Delta\right)
\end{aligned}
$$

where $\Delta$ is small.

The linearized system is $\dot{Z}=A Z$ with $A=\left[\begin{array}{cc}f^{\prime}\left(a_{1}+\Delta\right) / \delta & -1 / \delta \\ 1 & 0\end{array}\right]$. The eigenvalues of $A$ are $\lambda_{ \pm}=\frac{1}{2 \delta}\left(f^{\prime}\left(a_{1}+\Delta\right) \pm i \sqrt{4 \delta-f^{\prime 2}\left(a_{1}+\Delta\right)}\right) \cdot\left(a_{1}+\Delta, f\left(a_{1}+\Delta\right)\right)$ is an attracting (repulsive) focus if $\Delta>0(\Delta<0)$. We recover that $\left(a_{1}+\Delta, f\left(a_{0}+\Delta\right)\right)$ is stable when $\Delta>0$, unstable when $\Delta<0$. There is a limit cycle (periodic oscillations) when $a \in$ ]$a_{1}-\Delta, a_{1}[$ and by an analogous study also for $a \in] a_{0}, a_{0}+\Delta[$ for $\Delta>0$. This linear analysis is illustrated in Figure 4 at the end of the paper.

Let us now consider the following stochastic perturbation of FitzHugh-Nagumo system

$$
\begin{aligned}
\delta d X_{t} & =\left(-Y_{t}+f\left(X_{t}\right)\right) d t+\sqrt{\epsilon} d W_{t} \\
d Y_{t} & =\left(X_{t}-a\right) d t
\end{aligned}
$$

$\epsilon$ is a small parameter. The noise $\left(W_{t}\right)$ is a one dimensional brownian motion (or Wiener process): it is a Gaussian process with independent increments. Its trajectories are continuous but nowhere differentiable; however its time derivative can be given a meaning as a distribution and it is called white noise especially in engineering models. One can also study stochastic differential equations (cf. [10]). The noise $\left(W_{t}\right)$ induces transitions between the basins of attraction of two stable equilibrium points otherwise impossible when $\epsilon=0$. The celebrated theory of Large Deviations developed by Freidlin and Wentzell provides an estimation of the transition times among other results (cf. [5]). To give an idea of this theory we consider the following generic one dimensional situation. 
Consider the deterministic dynamical system

$$
d x_{t}=b\left(x_{t}\right) d t, \quad x_{0}=x
$$

and its stochastic perturbation

$$
d x_{t}^{\epsilon}=b\left(x_{t}^{\epsilon}\right) d t+\sqrt{\epsilon} d W_{t}, \quad x_{0}=x
$$

Assume that $x^{*}$ is the unique stable equilibrium of (4.7) and its basin of attraction is $D=] \alpha_{1}, \alpha_{2}$ [. Let us recall that $D$ is the set of points $x$ such that the solution of (4.7) starting at $x$ converges to $x^{*}$ when $t$ goes to infinity. If $x \in D,\left(x_{t}\right)$ never exits $D$.

Define $V(z)=-2 \int_{x^{*}}^{z} b(r) d r$, the quasi-potential with respect to $x^{*}$. If $V\left(\alpha_{1}\right)<V\left(\alpha_{2}\right)$ then, as $\epsilon \rightarrow 0$, for all $x \in D$, with probability 1 ,

- $\left(x_{t}^{\epsilon}\right)$ exits $D$ at $\alpha_{1}$

- exit time $\sim \exp \frac{V\left(\alpha_{1}\right)}{\epsilon}$

- $\left(x_{t}^{\epsilon}\right)$ has enough time to exit $D$ during $[0, T(\epsilon)]$ if $V\left(\alpha_{1}\right)<\epsilon \log T(\epsilon)$

For many more details and results we refer the reader to [5].

The slow-fast property of FitzHugh-Nagumo system allows to use large deviations in dimension 1 applied to the family of systems obtained when the slow variable is fixed to value $y$ :

$$
\delta d X_{t}=\left(-y+f\left(X_{t}\right)\right) d t+\sqrt{\epsilon} d W_{t}
$$

which we see as the stochastic perturbation of $\delta d x_{t}=\left(-y+f\left(x_{t}\right)\right) d t$. For each $y \in] f\left(a_{0}\right), f\left(a_{1}\right)$, the system $\delta d x_{t}=\left(-y+f\left(x_{t}\right)\right) d t$ has three equilibrium points $x_{-}^{*}(y)<x_{0}^{*}(y)<x_{+}^{*}(y)$ since $f$ is a cubic polynomial. $x_{ \pm}^{*}(y)$ are stable, $x_{0}^{*}(y)$ unstable. For each stable equilibrium consider its quasipotential $V_{ \pm}(y)=-2 \int_{x_{ \pm}^{*}(y)}^{x_{*}^{*}(y)}(-y+f(x)) d x$. In this bistable situation $V_{ \pm}(y)$ compare the depths of two wells with bottoms $x_{ \pm}^{*}(y)$. To decide when to switch from one well to the other, one must compare $V_{ \pm}(y)$ and $\frac{\epsilon}{\delta} \log \delta^{-1}$ This corresponds to the fact that the stochastic system (4.5)-(4.6) has three time scales: the slow one, the fast one and the scale of the noise given by $\epsilon$. The relative strenghts of $\delta$ and $\epsilon$ matters. One can show that when $\frac{\epsilon}{\delta} \log \delta^{-1} \rightarrow c$ (quasi-static regime cf. [4]) the trajectory of $\left(X_{t}, Y_{t}\right)$ may converges to a deterministic periodic trajectory when $t$ goes to infinity, even for values of $a$ strictly smaller than $a_{0}$. For details see [2]. Assume that $c$ is such that the Figure 3 holds. The periodic limiting trajectory is given by the union of the horizontal segments delimiting area $c / 2$ and the parts of the curve $y=f(x)$ joining these segments. One can also show that new bifurcation parameters appear due to noise (cf. [2]). For a general study of slow-fast systems perturbed by noise we refer the reader to [1].

Let us mention that the noise induced transitions described for the FitzHugh-Nagumo system are an example of the stochastic resonance phenomenon. This phenomenon can also explain the alternance of glacial eras of the earth. The mathematical study has been conducted by Herrmann and Imkeller [7]. Although the contexts of these two problems are different, the mathematical tool is for both the large deviations theory (cf. [5]) this is why we mention it briefly. The considered stochastic model is

$$
d X_{t}=\left(b\left(X_{t}\right)+f\left(\frac{t}{T}\right)\right) d t+\sqrt{\epsilon} d W_{t}
$$

where $f$ if a $T$-periodic function. The periodic perturbation is slow ( $T$ is large) and $\epsilon$ is small. The question is to find a noise intensity $\epsilon(T)$ which amplifies the periodic 


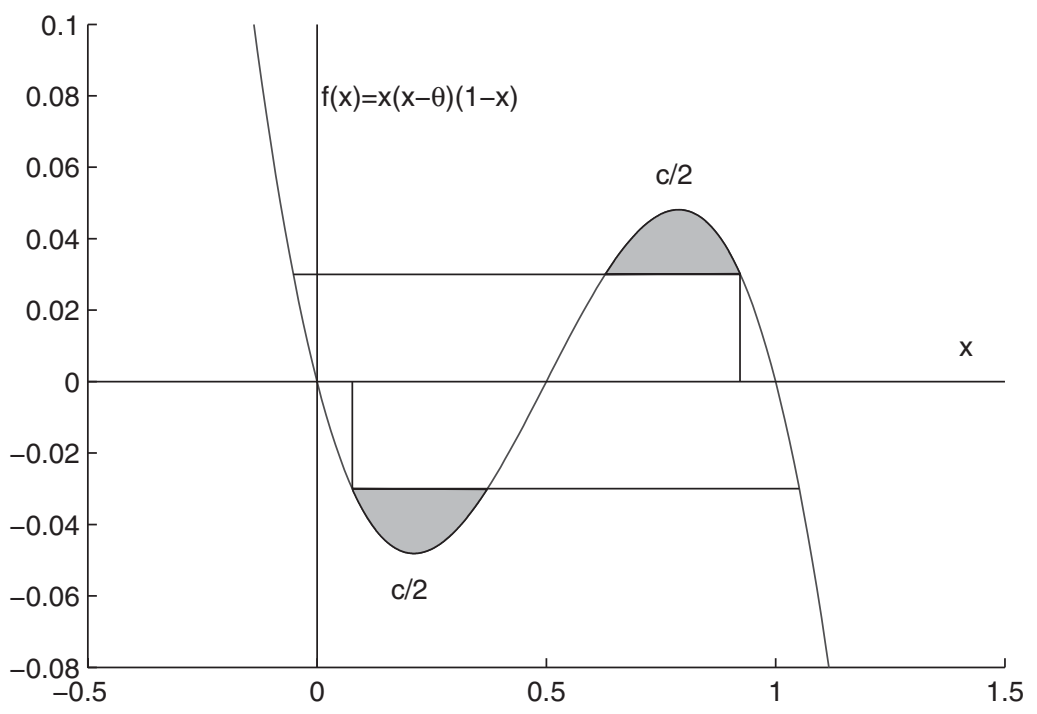

Figure 3. Periodic Transtitions Induced by Noise.
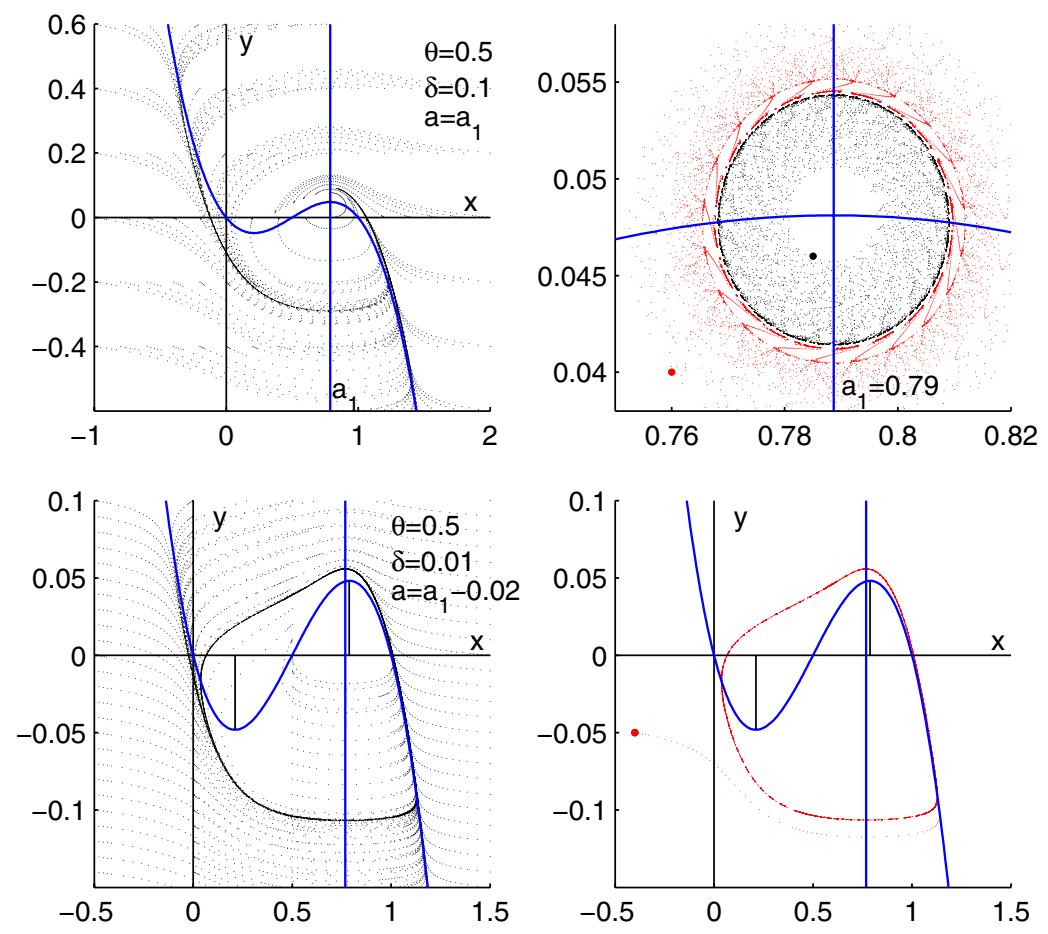

Figure 4. Stability Analysis of (4.1)-(4.2) around Bifurcation Parameter $a_{1}$. 
perturbation in a best possible way: this is stochastic resonance. We refer the reader to [7] and the references therein.

\section{References}

Berglund, N., Gentz, B., Noise-Induced Phenomena in Slow-Fast Dynamical Systems: A Sample Paths Approach. Springer (2006).

Doss, C., Thieullen, M., Oscillations and Random Perturbation of FitzHugh-Nagumo System. In preparation.

Ermentrout, G., B., Cowan, J., D., A Mathematical Thoery of Visual Hallucination Patterns. Biological Cybernetics, 34, 137-150 (1979).

Freidlin, M., I., Quasi-Deterministic Approximation, Metastability and Stochastic Resonance. Physica D 137, 333-352 (2000).

Freidlin, M., I., Wentzell, A., D., Random Perturbations of Dynamical Systems. Grundlehren der mathematischen Wissenschaften 260. Springer Verlag.

Gerstner, W., Kistler, W., Spiking Neuron Models. Single Neurons, Populations, Plasticity Cambridge University Press, Fourth Printing 2008.

Herrmann, S., Imkeller, P., Barrier crossings characterize stochastic resonance. Stochastics and Dynamics, 2 (3) 413-436 (2002).

Hodgkin, A., L., Huxley, A., F., A quantitative description of membrane current and its application to conduction and excitation in nerve. J. Physiol. (London) 117, 500-544 (1952).

Murray, J., D., Mathematical Biology. Biomathematics Texts. Springer Verlag. 2nd corrected edition.

Oksendal, B., Stochastic Differential equations: an introduction with applications. Universitext Springer 2003. 